\title{
Preparation of ZnS Modified PHBV Film by Chemical Bath Deposition Method
}

\author{
Haifeng Chen \\ Department of Materials chemistry \\ Huzhou University \\ Huzhou, China \\ headder20132931@qq.com
}

\author{
Guo lingmei \\ Department of Materials chemistry \\ Huzhou University \\ Huzhou, China \\ 705637226@qq.com
}

\begin{abstract}
Zinc nitrate hexahydrate, ethylene glycol, thiourea and triethanolamine were purchased to be starting materials. Nano zinc sulfide solution has been successfully prepared by Chemical bath deposition method, and then the prepared zinc sulfide modified polyhydroxybutyrate valerate (PHBV) to form the thin film. The morphology, structure, transparency and luminescent properties of the prototype were characterized by $\mathrm{X}$-ray diffraction, UV-visible spectrophotometer, polarized light microscopy, scanning differential thermal balance and fluorescence spectrophotometer. The results show that $\mathrm{ZnS}$ is the presence of a nucleating agent in the PHBV. With the zinc sulfide modified PHBV, the nucleation density and the toughness of the film can be improved while the composite film have fluorescence. But the addition of zinc sulfide can not change the crystal structure of PHBV films which will reduce the thermal stability of $\mathrm{PHBV}$ and inhibit the process of crystallization.
\end{abstract}

Keywords- ZnS; PHBV; chemical bath deposition method; fluorescence; thermal stability

\section{INTRODUCTION}

Zinc sulfide $(\mathrm{ZnS})$ is an important II-VI direct bandgap semiconductor material [1]. There are wurtzite and hexagonal crystal structure of $\mathrm{ZnS}$ with a wide direct band gap of from 3.6 to 3.8 ev[2-4]. As a result, it has wide band gap. Owing to its high refractive index, high transmittance and good photoelectric properties, it is widely used in various optical and optoelectronic devices, such as thin film electroluminescent (TFEL) display, ultraviolet and infrared light detector, a light-emitting diode (LED), solar cells. In addition, suffering from UV light irradiation and excitation of cathode ray and X-ray, the solid $\mathrm{ZnS}$ would produce radiation, which is a description of its fluorescent properties. U.Gangopadhyay [5] and his colleagues have successfully prepared $\mathrm{ZnS}$ film with low reflectivity ( an average of about $0.655 \%$ ) by CBD method, and the film is used as an anti-reflection layer of monocrystalline silicon cells, thus allowing the battery to get $13.8 \%$ the conversion efficiency. $\mathrm{ZnS}$ exhibits excellent performance in the electrical, optical, mechanical, magnetic and catalytic field, attracting more attention to research on nano-zinc sulfide.

There are many methods for preparing nano-ZnS particles currently. Chemical vapor deposition (CVD) [6-
7], sol gel method (Sol-gel) [8], atomic layer epitaxy (ALE) [9], thermal evaporation method [10-11], electrochemical deposition (ECD)[12-13] and pulsed laser deposition (PLD)[14] are the main chemical methods. And the $\mathrm{ZnS}$ which is used as a solar cell buffer layer is almost prepared by chemical bath deposition [15-17]. Polyhydroxybutyrate valerate (PHBV) is thermoplastic polyester which is synthesized by the fermentation of bacteria and microbial. And it is a biological material with starch being the main raw material, which is synthesized by fermentation engineering technology and exhibits excellent biodegradability, optical activity and biocompatibility [18]. PHBV have the biodegradability, good biocompatibility, bio-absorptive melting point, besides, it shows the high optical transparent, along with gap structure, which is beneficial for all kinds of catalyst reaction and carrier recovery.

Here, we adopt the zinc sulfide solution prepared by the chemical bath deposition [19] to modify PHBV to form thin films. The morphology, structure, transparency and luminescent properties of the prototype were characterized by X-ray diffraction, UV-visible spectrophotometer, polarized light microscopy, scanning differential thermal balance and fluorescence spectrophotometer.

\section{EXPERIMENTAL AND CHARACTERIZATION}

First, $0.561 \mathrm{~g}$ of the zinc nitrate hexahydrate weighed by an electronic balance and $60 \mathrm{ml}$ of distilled water taken by a measuring cylinder were added into a $200 \mathrm{ml}$ of clean beaker, and then put a magneton as well. While stirring at the thermostatic magnetic mixer for $10 \mathrm{~min}$ until it was completely dissolved, $10 \mathrm{ml}$ triethanolamine (by measuring cylinder) was slowly added into the above solution to obtain a mixture. $0.228 \mathrm{~g}$ of thiourea and then $80 \mathrm{ml}$ of ethylene glycol taken by measuring cylinder were placed into the solution, and stirred uniformly. Finally, the resulting solution was drained into 3 mouth flask by a glass rod. Turned on the heating jacket and set the temperature to $180{ }^{\circ} \mathrm{C}$, opened the reflux device, heated the solution 3-4 hours to complete reaction, then nano zinc sulfide solution was got. Preparation of loading $\mathrm{ZnS}$ modified PHBV film: added $0.2 \mathrm{~g}$ of PHBV taken by a measuring cylinder into a $25 \mathrm{ml}$ of beaker, dissolved it in 
$8 \mathrm{ml}$ chloroform, and then put $1.194 \mathrm{ml}$ of the zinc sulfide solution into the beaker by pipette and stirred at the thermostatic magnetic mixer for $0.5 \mathrm{~h}$; when completely dissolved, the compound of $1 \%$ zinc sulfide and PHBV can be obtained after homogeneous mixing. Then dried the composite solution at room temperature in the hood, so the loading nano $\mathrm{ZnS}$ modified PHBV thin film with photocatalytic activity was got. According to the same method, $2 \%, 3 \%, 4 \%, 5 \%$ of the zinc sulfide PHBV thin film was got. The chemical reagents involved were analytically pure. When the film above were prepared, XRD patterns of the samples were measured on XD- 6 of Bejing Puxi apparatus Co-company; UV visible spectroscopy was obtained from Evolution 220 UV visible spectrophotometry of American Thermo Electron Corporation; the TG of the sample was got by CRY-2P thermogravimetric analyzer of Bejing Hengjiu scientific instrument factory; the fluorescent properties were tested by RF-5301PC fluorophotometer of SHIMADZU; microscopic hot stage temperature control device of Shanghai Changfang Optical Instrument Co-company pressed tablets at $170{ }^{\circ} \mathrm{C}$, and compared them with high pure $\mathrm{PHBV}$, then observed the crystalline morphology by polarizing microscope and compared the size of spherulites.

\section{RESULTS AND DISCUSSION}

\section{A. The effect of ZnS doping amount on the crystal type of composite PHBV films}

The curve in Fig. 1 presents the XRD pattern of PHBV film modified by $\mathrm{ZnS}$, pure PHBV powder, pure PHBV film and $\mathrm{ZnS}$. As shown in the figure, the diffraction range of $\mathrm{X}$ ray diffraction spectrogram is 10 to 80 . The $\mathrm{X}$ ray diffraction peaks of $\mathrm{ZnS}$ in the picture is roughly consistent with the standard characteristic peaks of $\beta-\mathrm{ZnS}$ (JCPDS-5-556), which showed the prepared samples are $\mathrm{ZnS}$ microcrystals that belong to a face-centered cubic lattice and have zinc-blende structure. The spectra of $\mathrm{ZnS}$ also manifested that the diffraction peaks have obvious width and the growth of the crystal is incomplete. And what can also be seen in the figure is that the X-ray diffraction patterns of pure PHBV film, pure PHBV powder and PHBV modified by $\mathrm{ZnS}$ is roughly the same.

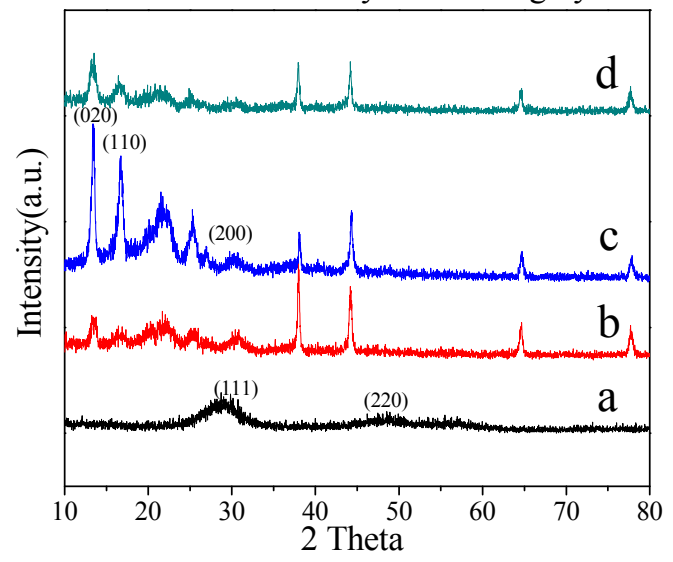

Figure 1. XRD patterns of samples. (a: ZnS; b: PHBV film; c: PHBV powder; d: PHBV films modified with $\mathrm{ZnS}$ )
$\mathrm{ZnS}$ diffraction pattern in the figure shows that the $\mathrm{ZnS}$ is amorphous. So the presence of $\mathrm{ZnS}$ in PHBV film exerts no evident influence on the position of diffraction peak of the PHBV film and the form, there is only one crystalline phase. When PHBV powder was manufactured into film, the intensity of (020) and (110) face decreased. Although the presence of $\mathrm{ZnS}$ in PHBV film can not change the position of diffraction peak of the PHBV film and the form, the intensity of (020) and (110) face has been enhanced. So, the results above indicate that the presence of $\mathrm{ZnS}$ in PHBV film doesn't change the crystalline morphology of PHBV while exerting some effect on the crystallization strength of certain direction.

\section{B. The effect of ZnS dosage on the crystalline of composite PHBV films}

Fig. 2 was the polarization microscope photographs of PHBV films with different content of $\mathrm{ZnS}$ and the pure PHBV. Polarizing analysis is so important that we can know the impact of $\mathrm{ZnS}$ on the PHBV, which plays a crucial guiding role in improving the mechanical properties of the material. Seen from the chart, the sizes of the spherulites are different, and all of them have black cross extinction phenomenon. Fig. 2(e) is a picture of pure PHBV crystalline. In the picture, the spherulites are complete but rare. The other five pictures are polarizing figures of PHBV films modified with $1 \%, 2 \%$, $3 \%, 4 \%$ and $5 \%$ of the $\mathrm{ZnS}$ respectively. Compared with the pure PHBV samples, the number of the spherulites increases obviously and the size of the spherulites is smaller than pure PHBV. Because nano $\mathrm{ZnS}$ is acting as a nucleating agent, after adding it in the PHBV, the nucleation density is higher and the crystallization speed is quicker than the pure PHBV at the same crystallization temperature. In the Fig. 2 (a) to (e), when adding $1 \%$ of the $\mathrm{ZnS}$, the nucleation density is smaller than $2 \%$.

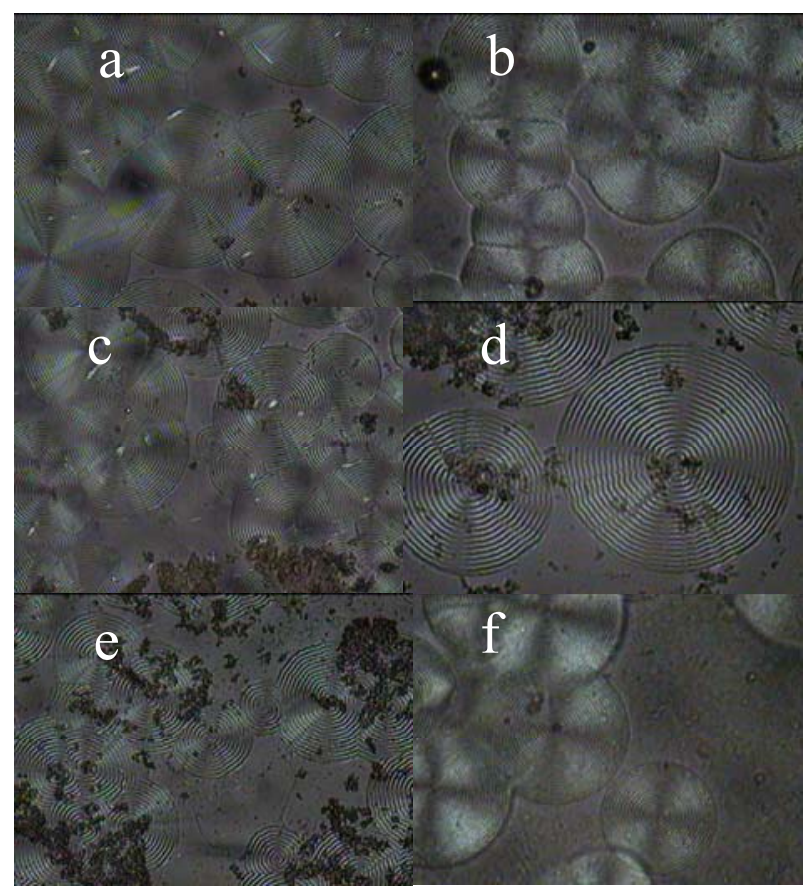

Figure 2. The polarization microscope photographs of PHBV films with different content of $\mathrm{ZnS}$ and the pure PHBV: (a) $1 \% \mathrm{ZnS}$; (b) $2 \%$ $\mathrm{ZnS}$; (c) $3 \% \mathrm{ZnS}$; (d) $4 \% \mathrm{ZnS}$; (e) $5 \% \mathrm{ZnS}$; (f) the pure PHBV. 
When adding $3 \%$ and $4 \%$ of the $\mathrm{ZnS}$, the nucleation density has no obvious change. So $\mathrm{ZnS}$ has given full play to its role of a nucleating agent when added a small number into the PHBV film, and excessive addition exerts little effect on the nucleation of the PHBV film. The five pictures have a phenomenon that the spherulite size is not the same, which is because the space is so compressed that it loses its original shape when the spherulites grow to contact each other. The spherulite nucleation order is different in the same region, and the spherulites are smaller when there are many crystal nuclei [20]. The spherulite of pure PHBV is very big and perfect with high brittleness, the spherulite size of the pure PHBV decreases and the nucleus density of the spherulite enhances after the addition of $\mathrm{ZnS}$, therefore $\mathrm{ZnS}$ not only plays the role of nucleating agent, but also makes the sample strength increase, thus the toughness increases

\section{The effect of $\mathrm{ZnS}$ dosage on the thermal performance of composite PHBV films}

Fig. 3 is the thermogravimetric analysis curve of the pure PHBV film and the PHBV thin film material with $1 \%$ and $5 \%$ of the $\mathrm{ZnS}$. The experiments were carried out at the same heating rate (10 degrees $/ \mathrm{min})$, as can be seen from the chart, these substances have only one weight loss and the temperature is between $200{ }^{\circ} \mathrm{C}-300{ }^{\circ} \mathrm{C}$ roughly. The weight loss temperature of pure PHBV film is higher than that of PHBV thin films modified with $\mathrm{ZnS}$. And the weightlessness end temperature of the pure PHBV is obviously higher than that of the PHBV thin films modified with $\mathrm{ZnS}$. The weight loss temperature of the PHBV thin film modified with $1 \%$ of $\mathrm{ZnS}$ is higher than that of other addition. In the picture, the thermal stability of the PHBV thin film modified with $5 \%$ of $\mathrm{ZnS}$ is evidently poorer than that of other addition, and its TGA termination temperature is relatively low, while the PHBV thin films with other addition have TGA termination temperatures with little difference. As a result, doping zinc sulfide imposes little influence on modifying the heat stability of the pure PHBV film, and on the contrary, it reduces the thermal stability. If adding zinc sulfide is a must, it will be better to lower the zinc sulfide content.

Fig. 4 is the melting analysis curve of the pure PHBV film and the PHBV thin film material with $1 \%$ to $5 \%$ of $\mathrm{ZnS}$. In the curve of PHBV, the first peak is the binary eutectic melting peak of $\mathrm{HV}$ and $\mathrm{HB}$, the second peak is the melting peak of $\mathrm{HB}$, and both are endothermic peak. In the figure, the first melting peak temperature of the pure PHBV film is $300{ }^{\circ} \mathrm{C}$, and the first melting peak temperature moves forward for about $300{ }^{\circ} \mathrm{C}$ after adding the $\mathrm{ZnS}$, which indicates that the crystal transition of the $\mathrm{HV}$ and HB binary eutectic of the PHBV speeds up when modified with $\mathrm{ZnS}$. The peak size changes as well. The two peak areas of the thin film modified with $\mathrm{ZnS}$ are smaller than that of the pure PHBV, indicting that the zinc sulfide reduces the thermal effect of PHBV. The melting peak of the thin film modified with $\mathrm{ZnS}$ moves forward and the peak area reduces obviously when compared with the pure PHBV film. When the content of zinc sulfide was $4 \%$ and $5 \%$, the second peak of the film is very small, next to nothing, which shows that adding a small amount of $\mathrm{ZnS}$ could inhibit the crystallization process of the PHBV.

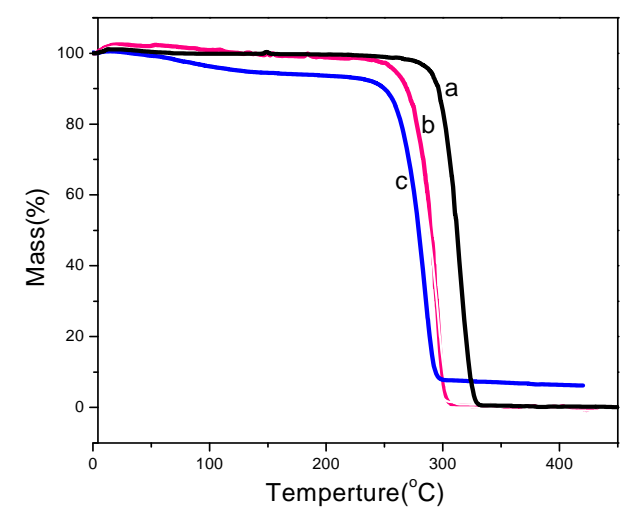

Figure 3. the thermogravimetric analysis curve of the pure PHBV film (a), the $1 \% \mathrm{ZnS}$ modified PHBV film(b) and the $5 \% \mathrm{ZnS}$ modified PHBV film(c).

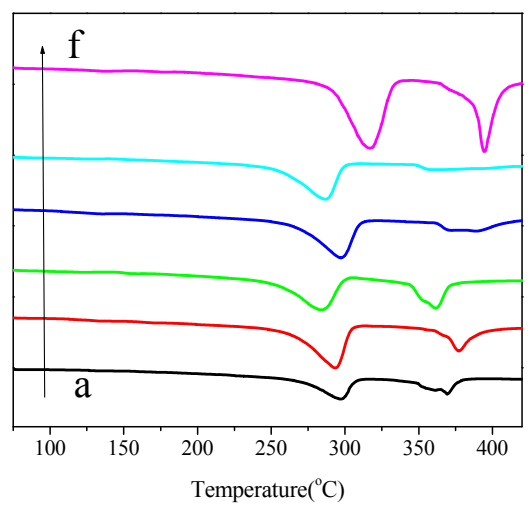

Figure 4. the melting analysis curve of films.(a to e: $1 \%$ to $5 \%$ of $\mathrm{ZnS}$ into PHBV films; f: pure PHBV film).

\section{The effect of ZnS dosage on the optical absorption properties of composite PHBV films}

Fig. 5 is the ultraviolet spectrum analysis curve of the pure PHBV and the PHBV thin film containing $1 \%$ to $5 \%$ of $\mathrm{ZnS}$. The ultraviolet spectra line of the pure PHBV is of nonlinear growth, and the other thin film curve containing zinc sulfide decreases nonlinearly. So it is obviously that the transparency of pure PHBV film is higher than that of the film modified with $\mathrm{ZnS}$. But the rate of change of the pure PHBV transparency decreases as the wavelength increases, while the rate of change of the film with $1 \%$ and $2 \%$ of $\mathrm{ZnS}$ increases with increasing wavelength.

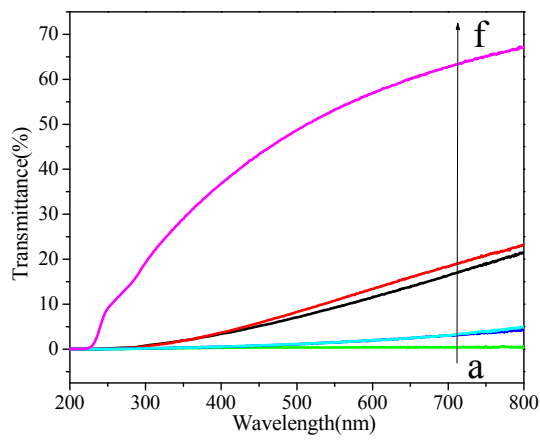

Figure 5. UV-visible spectrums of the films.(a to e: $5 \%$ to $1 \%$ of $\mathrm{ZnS}$ into PHBV films; f: pure PHBV film). 
And the ultraviolet spectra curve is almost parallel to the $\mathrm{X}$ axis and the transparency is close to zero when the film contains $3 \%$ and $4 \%$ of $\mathrm{ZnS}$. So doping $\mathrm{ZnS}$ exerts impact on the transparency of the PHBV film. Zinc sulfide reduces the transparency of the PHBV film, which may has to do with the fail of homogeneous mixing with chloroform when preparing the composite thin film.

\section{CONCLUSION}

In this paper, nano zinc sulfide solution had been successfully prepared by Chemical bath deposition method. According to different proportion, the PHBV was filled with the prepared $\mathrm{ZnS}$ to form composite films. The research and analysis focused on the crystal structure, crystalline properties, fluorescence, TGA performance and other properties of obtained blends, and the understanding of the impact of $\mathrm{ZnS}$ on the PHBV. Thus we have obtained the following conclusions. It showed that the location and the form of the X-ray diffraction peaks of the pure PHBV containing $\mathrm{ZnS}$ is similar to the pure PHBV, only the intensity of the peak changes. The addition of the $\mathrm{ZnS}$ does not affect the crystal structure of the pure PHBV. After adding $\mathrm{ZnS}$ to form composite film, the presence of $\mathrm{ZnS}$ can reduce the thermal effect of pure PHBV and inhibit the crystallization process. $\mathrm{ZnS}$ has played the role of nucleating agent in PHBV films and increases the toughness of PHBV.

\section{ACKNOWLEDGMENT}

This work was financially supported by scientic research project of Huzhou University (2015XJKY31).

\section{REFERENCES}

[1] Dunqing Wang, Xiuling Jiao, Dairong Chen, "The outline of the Zinc sulfide properties, applications and preparation methods," Shandong chemical industry, vol. 32, 2003, pp. 12-15.

[2] Fang X, Zhai T, Gautam U K, et al, "ZnS nanostructures: From synthesis to applications," Progress Materials Science, vol. 56, 2011,pp. 175-287, doi: 10.1016/j.pmatsci.2010.10.001

[3] Ruzhang Ma, Minghua Jiang, Zuxiong $\mathrm{Xu}$, Introduction to Functional Materials, 1st ed. Beijing Metallurgical Industry Press, 1999, pp.33-37

[4] Dunqing Wang, Xiuling Jiao, Dairong Chen, "The outline of the Zinc sulfide properties, applications and preparation methods," Shandong chemical industry, vol. 32, 2003, pp. 12-15

[5] U. Gangopadhyay, Kyunghae Kim, D. Mangalaraj, "Low cost CBD ZnS antireflection coating on large area commercial nanocrystalline silicon solar cells," Applied Surface Science, vol. 230, 2004, pp. 364-370, doi: 10.1016/j.apsusc.2004.02.059
[6] V. Karaksina E, A. Gracheva T, N. Shevarenkov D, "Structural defects in CVD ZnS," Inorganic Materials, vol. 46, 2010, pp. 6-10, doi: 10.1134/S0020168510010024

[7] Yao-yuan Y, Yi-chao C, Yan-ping D, et al, "Effect of Technical Parameters on Deposition Rate of CVD ZnS," Journal of Synthetic Crystals, vol. 33, 2004, pp. 238-240

[8] Goktas A, Aslan F, Yasar E, et al, "Preparation and characterisation of thickness dependent nano-structured $\mathrm{ZnS}$ thin films by sol-gel technique," Journal of Materials Science: Materials in Electronics, vol. 23, 2012, pp. 1361-1366, doi: 10.1007/s10854-011-0599-z

[9] Tanninen V, Oikkonen M, O. Tuomi T, "X-Ray Diffraction Study of Thin Electroluminescent ZnS Films Grown by Atomic Layer Epitaxy,” Physica Status Solidi (a ), vol. 67,2006, pp. 573-583, doi: 10.1002 pssa.2210670227

[10] Shaban S M, Saeed N M, Al-Haddad R M S, "Fabrication and study zinc sulfide schottky barrier detectors," Indian Journal of Science \& Technology, vol. 4, 2011, pp. 384-386, doi: 10.17485/ijst/2011/v4i4/30006

[11] Li Q, Wang C, "Fabrication of wurtzite $\mathrm{ZnS}$ nanobelts via simple thermal evaporation," Applied physics letters, vol. 83, 2003, pp. 359-361, doi: 10.1063/1.1591999

[12] Fathy N, Kobayashi R, Ichimura M, "Preparation of ZnS Thin Films by the Pulsed Electrochemical Deposition," Materials Science and Engineering: B, vol. 107,2004, pp. 271-276, doi: 10.1016/j.mseb.2003.11.021

[13] Xu X, Fei G, Yu W, et al, "Preparation and formation mechanism of $\mathrm{ZnS}$ semiconductor nanowires made by the electrochemical deposition method," Nanotechnology, vol. 17, 2006, pp. 426-429, doi: 10.1088/0957-4484/17/2/013

[14] Yano S, Schroeder R, Sakai H, et al, "High-electric-field photocurrent in thin-film $\mathrm{ZnS}$ formed by pulsed-laser deposition," Applied Physics Letters, vol. 82, 2003, pp. 2026 - 2028, doi: 10.1063/1.1564287

[15] Q. Bian Z, B. Xu X, B. Chu j, et al, "Study of chemical bath deposition of $\mathrm{ZnS}$ thin films with substrate vibration," Surface Review and Letters (SRL), vol. 15, 2008, pp. 821-827, doi: 10.1142/S0218625X08012098

[16] Sartale S, Sankapal B, Lux M, "Preparation of nanocrystalline ZnS by a new chemical bath deposition route," Thin Solid Films, vol. 480, 2005, pp. 168-172, doi: 10.1016/j.tsf.2004.11.054

[17] KJ H, P R, CM. L, "Chemical bath deposition synthesis of submicron ZnS-coated polystyrene," Journal of Colloid and Interface Science, vol. 308, 2007, pp. 112-120, doi: 10.1016/j.jcis.2006.11.057

[18] Baotong Hao, Baolin Liu, "The cryopreservation of applied nanoparticles in cells," Chinese Clinical Rehabilitative Tissue Engineering Research, vol. 12, 2008, pp. 8140-8142

[19] Dona J M, Herrero J, "Process and film characterization of chemical-bath-deposited ZnS thin films," Journal of the Electrochemical Society, vol. 141, 1994, pp. 205-210, doi: $10.1149 / 1.2054685$

[20] Faxin Dong, Zhenjun Wang, Yingqi Jia, "The method of UV spectrophotometer reflectance spectroscopy," Physical Testing and Chemical Analysis Part, vol. 39, 2003, pp. 319-320 